\title{
Photoluminescence-excitation spectroscopy as a highly sensitive probe for carrier transport processes affected by surface damages in $\mathrm{Al}_{\mathrm{x}}$ Ga1-x N/GaN heterostructures
}

Hideo Takeuchi, Yoshitsugu Yamamoto, Yoshitaka Kamo, Tetsuo Kunii, Tomoki Oku, Takeo Shirahama, Hiroyasu Tanaka, Masaaki Nakayama

\begin{tabular}{|c|l|}
\hline Citation & Journal of Applied Physics, 102(4);043510 \\
\hline Issue Date & 2007-08-22 \\
\hline Type & Journal Article \\
\hline Textversion & Publisher \\
\hline Right & $\begin{array}{l}\text { C 2007 American Institute of Physics. This article may be downloaded for personal } \\
\text { use only. Any other use requires prior permission of the author and AIP Publishing. } \\
\text { The following article appeared in Journal of Applied Physics and maybe found at } \\
\text { https://doi.org/10.1063/1.2769776 }\end{array}$ \\
\hline DOI & $10.1063 / 1.2769776$ \\
\hline
\end{tabular}

Self-Archiving by Author(s)

Placed on: Osaka City University Repository

Hideo Takeuchi, Yoshitsugu Yamamoto, Yoshitaka Kamo, Tetsuo Kunii, Tomoki Oku, Takeo Shirahama, Hiroyasu Tanaka, Masaaki Nakayama. (2007). Photoluminescence-excitation spectroscopy as a highly sensitive probe for carrier transport processes affected by surface damages in AlxGa1-xN/GaN heterostructures. Journal of Applied Physics. 102, 43510. 


\title{
Photoluminescence-excitation spectroscopy as a highly sensitive probe for carrier transport processes affected by surface damages in $\mathrm{Al}_{x} \mathrm{Ga}_{1-x} \mathrm{~N} / \mathrm{GaN}$ heterostructures
}

\author{
Hideo Takeuchi, ${ }^{a)}$ Yoshitsugu Yamamoto, Yoshitaka Kamo, Tetsuo Kunii, and Tomoki Oku \\ $R \& D$ Department, High Frequency and Optical Device Works, Mitsubishi Electric Corporation, \\ 4-1 Mizuhara, Itami, Hyogo 664-8641, Japan
}

\section{Takeo Shirahama}

Wafer Manufacturing Department, High Frequency and Optical Device Works, Mitsubishi Electric Corporation, 4-1 Mizuhara, Itami, Hyogo 664-8641, Japan

\author{
Hiroyasu Tanaka and Masaaki Nakayama \\ Department of Applied Physics, Graduate School of Engineering, Osaka City University, \\ 3-3-138 Sugimoto, Sumiyoshi-ku, Osaka 558-8585, Japan
}

(Received 17 April 2007; accepted 29 June 2007; published online 22 August 2007)

\begin{abstract}
We demonstrate that photoluminescence-excitation (PLE) spectroscopy can probe with high sensitivity the effects of plasma-induced surface damages on photogenerated-carrier-transport processes in $\mathrm{Al}_{x} \mathrm{Ga}_{1-x} \mathrm{~N} / \mathrm{GaN}$ heterostructures, on the basis of systematic optical and structural characterization results for the as-grown reference sample and the plasma-exposed sample. It is found from the structural characterizations with atomic force microscopy that the plasma exposure remarkably modifies the atomic step boundaries and the pits on the $\mathrm{Al}_{x} \mathrm{Ga}_{1-x} \mathrm{~N}$ surface, which leads to a remarkable difference between the PLE spectra of the bound exciton photoluminescence from the underlying GaN layer in the two samples. The PLE spectrum of the reference sample shows a step rising from the $\mathrm{Al}_{x} \mathrm{Ga}_{1-x} \mathrm{~N}$ fundamental transition energy toward the high energy side, whereas the rising step disappears in the PLE spectrum of the plasma-exposed sample. In contrast, the reflectance characteristics are the same in the two samples; i.e., the excitonic transition itself is not influenced by the plasma exposure. The present findings indicate that the PLE spectral profile is sensitive to the change in efficiency of the photogenerated carrier injection from the $\mathrm{Al}_{x} \mathrm{Ga}_{1-x} \mathrm{~N}$ layer to the GaN layer. Thus, it is concluded that the PLE characterization is effective to probe the photogenerated-carrier transport in heterostructures. (C) 2007 American Institute of Physics.
\end{abstract}

[DOI: $10.1063 / 1.2769776]$

\section{INTRODUCTION}

In the field of semiconductors science and technology, it is essential to characterize surfaces, because material properties and device characteristics are sensitive to the surface conditions. There have been numerous reports on this issue. It is worth noting that the surface conditions can dominate electronic properties. ${ }^{1,2}$ For example, several research groups clarified the relation between the surface treatment and surface recombination velocity, ${ }^{2-4}$ where the surface recombination velocity is a parameter peculiar to the carrier recombination process at the surface. ${ }^{2}$ The characterization of surfaces is, therefore, indispensable for the electronic and/or optical device fabrication, processes of which often introduce damages to the surface of devices and modify the carrier-transport processes leading to changes in device characteristics. In fact, the relation between the device characteristics and surface conditions has still been extensively studied. For instance, in $\mathrm{Al}_{x} \mathrm{Ga}_{1-x} \mathrm{~N} / \mathrm{GaN}$-based high-electronmobility transistors (HEMTs), which have been attracting much attention for high power microwave devices, ${ }^{5,6}$ gate leakage currents are increased by the surface defects in the

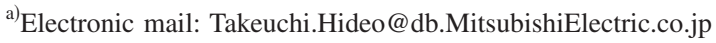

$\mathrm{Al}_{x} \mathrm{Ga}_{1-x} \mathrm{~N}$ Schottky layer. ${ }^{7}$ In addition, it is considered that rf characteristics of $\mathrm{Al}_{x} \mathrm{Ga}_{1-x} \mathrm{~N} / \mathrm{GaN}$ HEMTs are influenced by the surface traps. ${ }^{8,9}$

It is desirable that the effects of the surface damages should be systematically evaluated in the following procedure. The first step is to characterize the modification of the surface structure. The structural characterizations are, in general, performed with the aid of microscopical techniques such as atomic force microscopy (AFM), which provides information on morphological profiles. Next, it becomes important to clarify the relation between the structural characteristics and the carrier-transport processes. From the viewpoint of contactless and convenient characterizations, optical spectroscopic measurements are suitable. Photoluminescence (PL) spectroscopy is usually used as a probe for crystal quality influencing carrier transports; however, it is difficult to observe the PL signals from the barrier layer in heterojunctions, e.g., $\mathrm{Al}_{x} \mathrm{Ga}_{1-x} \mathrm{~N}$ layers in $\mathrm{Al}_{x} \mathrm{Ga}_{1-x} \mathrm{~N} / \mathrm{GaN}$ heterostructures, because, before the radiative recombination in the barrier layers, photogenerated carriers and excitons rapidly flow into an adjacent layer with a lower potential energy. Accordingly, in the case where the above-mentioned difficulty is prominent, it is required to apply a spectroscopic measurement that can sensitively probe the photogenerated 
carriers injected from the barrier layers to the adjacent layer. Here, we focus our attention to photoluminescenceexcitation (PLE) spectroscopy as the suitable method. In the PLE measurement, the intensity of a given PL band is recorded as a function of excitation photon energy. It is usually recognized that the PLE spectrum exhibits an absorption-like profile. ${ }^{10}$ The PLE spectrum, therefore, reflects the absorption characteristics of the luminescent layer. The PLE spectrum also contains the information on photogeneratedcarrier-transport processes in the heterostructures because the PL signal from a layer with the lowest potential is affected by the injection of the photogenerated carriers from higherpotential layers, which suggests that the PLE measurements are applicable to probe the photogenerated-carrier transport in the heterostructures. The conventional PLE characterization has been mainly taking account of the similarity between the absorption and PLE spectra, whereas it has paid little attention to the sensitivity of the PLE spectroscopy to the carrier transport. Accordingly, in order to utilize the PLE measurement as a probe for the effects of the surface damages on the carrier transport, it is necessary not only to systematically compare the PLE characteristics with the surface damages but also to reconsider the nature of the PLE spectroscopy from the viewpoint of semiconductor physics.

In the present work, we have explored the feasibility of applying the PLE measurement to probe the effects of surface damages on photogenerated-carrier-transport processes from the $\mathrm{Al}_{x} \mathrm{Ga}_{1-x} \mathrm{~N}$ layer to the $\mathrm{GaN}$ layer in $\mathrm{Al}_{x} \mathrm{Ga}_{1-x} \mathrm{~N} / \mathrm{GaN}$ heterostructures. We describe a wide range of optical and structural characteristics of the two samples, an as-grown reference sample and a sample exposed to the plasma, for the purpose of demonstrating the nature of the PLE spectroscopy as well as the relation between the PLE characteristics and surface damages. The present article is organized as follows. At first, we evaluate the effects of the plasma exposure on the surface morphology of the $\mathrm{Al}_{x} \mathrm{Ga}_{1-x} \mathrm{~N}$ top layer by means of grazing incidence $\mathrm{x}$-ray reflection (XRR) (Ref. 11) and AFM measurements, where the XRR patterns are sensitive both to the layer thickness and to the surface damages causing roughness. The surface damages are microscopically observed as AFM images, which reveal the characteristics of the surface damages induced by the plasma exposure. Next, we characterize the samples using PL spectroscopy for the following three purposes. The first purpose is to determine the detection energy for the PLE measurement. The second purpose is to obtain the information required for the assignment of the optical signals, e.g., a compressive biaxial strain, which often exists in $\mathrm{GaN}$ epitaxial layers grown on sapphire substrates and influences the optical properties. ${ }^{12}$ The third purpose is to verify that the plasma exposure does not affect the underlying GaN layer. Finally, we investigate how the surface damages observed by the structural characterization influence the PLE and reflectance spectra. The reflectance spectrum of the plasma-exposed sample shows a clear pattern resulting from the excitonic transitions in the $\mathrm{Al}_{x} \mathrm{Ga}_{1-x} \mathrm{~N}$ layer, which is the same pattern as that of the reference sample; namely, the reflectance characteristics of the excitonic transition are hardly affected by the surface damages. In contrast, the PLE spectra of the bound exciton luminescence from the $\mathrm{GaN}$ layer exhibit a remarkable difference. The PLE spectrum of the reference sample shows a step rising from the fundamental transition energy of $\mathrm{Al}_{x} \mathrm{Ga}_{1-x} \mathrm{~N}$ toward the high energy side, whereas the rising step disappears in the PLE spectrum of the plasma-exposed sample. We discuss the origin of the disappearance of the rising step in the PLE spectrum of the plasma-exposed sample from the viewpoint of carriertransport properties, taking account of the difference in spectroscopic nature between the PLE and reflectance.

\section{SAMPLES AND EXPERIMENTAL PROCEDURES}

The examined $\mathrm{Al}_{x} \mathrm{Ga}_{1-x} \mathrm{~N} / \mathrm{GaN}$ heterostructure was grown on a $c$-plane sapphire substrate by metal organic vapor phase epitaxy. The epitaxial layer sequence was designed as follows from the top: a $27 \mathrm{~nm}$ thick undoped $\mathrm{Al}_{0.2} \mathrm{Ga}_{0.8} \mathrm{~N}$ layer and a $2.2 \mu \mathrm{m}$ thick undoped GaN layer. After the epitaxial growth, the substrate with the $\mathrm{Al}_{0.2} \mathrm{Ga}_{0.8} \mathrm{~N}(27 \mathrm{~nm}) / \mathrm{GaN}(2.2 \mu \mathrm{m})$ heterostructure was cut into pieces. One of the pieces, Sample No.1, was kept as a reference sample, while another piece, Sample No. 2, was exposed to the plasma consisting of $\mathrm{Cl}_{2}$ and $\mathrm{O}_{2}$ gases at the total pressure below $1 \mathrm{~Pa}$. The purpose of the plasma exposure was only to cause the surface damage, so that, in order to minimize the reduction of the $\mathrm{Al}_{0.2} \mathrm{Ga}_{0.8} \mathrm{~N}$-layer thickness, the exposure time was limited within $1 \mathrm{~min}$. In addition, the mixture ratio of $\mathrm{Cl}_{2}$ to $\mathrm{O}_{2}$ was kept below 10. The XRR measurement was performed at room temperature using a $\mathrm{Cu} K \alpha 1$ line as a probe beam. The AFM observation was also carried out at room temperature with a tapping mode. The PLE and reflectance spectra of the two samples were measured at a temperature of $10 \mathrm{~K}$. The probe beam was monochromatic light with a spectral width of $0.4 \mathrm{~nm}$. In the PLE measurement, the detection energy was tuned within the energy range of the excitonic PL from the GaN layer because the present experiments were intended to detect the signal resulting from the photogenerated carriers escaping from the $\mathrm{Al}_{0.2} \mathrm{Ga}_{0.8} \mathrm{~N}$ layer to the $\mathrm{GaN}$ layer. In order to determine the detection energy, the PL spectra were measured in advance at $10 \mathrm{~K}$. The excitation beam source for the PL was provided from a He-Cd laser. The photon energy and power density of the excitation beam were $3.815 \mathrm{eV}$ and $10 \mathrm{~mW} / \mathrm{cm}^{2}$, respectively.

\section{EXPERIMENTAL RESULTS AND DISCUSSION}

\section{A. Effects of the plasma exposure on the surface structure of the $\mathrm{Al}_{0.2} \mathrm{Ga}_{0.8} \mathrm{~N}$ layer}

Figure 1 shows the XRR patterns of Sample No. 1 and No. 2 as a function of glancing angle $\theta$. The position of the dashed-and-dotted line corresponds to the critical angle $\theta_{c}$ of the $\mathrm{Al}_{0.2} \mathrm{Ga}_{0.8} \mathrm{~N}$ layer calculated with use of the equations ${ }^{11}$

$$
\theta_{c}=\sin ^{-1} \sqrt{2 \delta},
$$




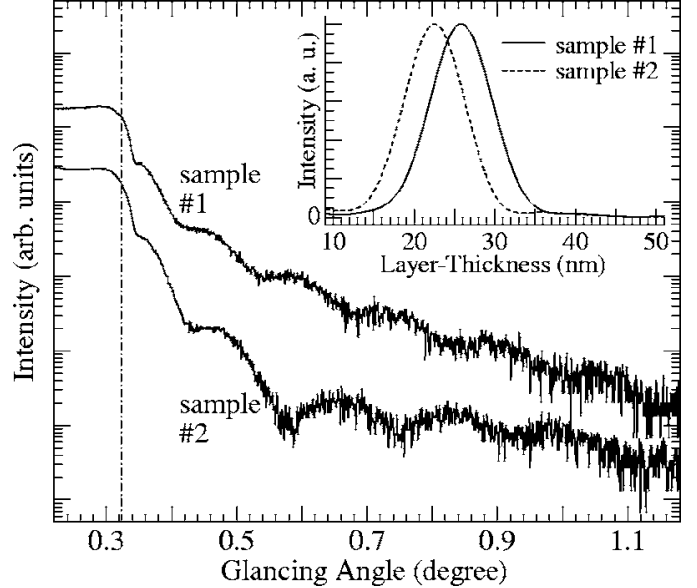

FIG. 1. XRR patterns of Sample No. 1 and No. 2 as a function of glancing angle. For clarity, each pattern is vertically shifted. Dashed-and-dotted line: position of the critical angle for $\mathrm{Al}_{0.2} \mathrm{Ga}_{0.8} \mathrm{~N}$. Inset: FT power spectra of Sample No. 1 and No. 2 as a function of layer thickness.

$$
\delta=\frac{N_{A} r_{e} \lambda^{2} \rho\left(Z+f^{\prime}\right)}{2 \pi M},
$$

where $N_{A}, r_{e}$, and $\lambda$ are the Avogadro number, the classical electron radius, and the wavelength of the $\mathrm{x}$ ray, respectively, and $\rho$ is the density of material. The value of $\rho$ is $5.566 \mathrm{~g} / \mathrm{cm}^{3}$ for $\mathrm{Al}_{0.2} \mathrm{Ga}_{0.8} \mathrm{~N} .{ }^{13}$ The quantities $Z, f^{\prime}$, and $M$ are the averages of the atomic number, of the anomalous scattering factor, and of the atomic mass, respectively, where the averages are calculated using the fraction of each constituent atom as a weighting factor. In the derivation of $f^{\prime}$, the anomalous scattering factors employed were 0.212, -1.281 , and 0.031 for $\mathrm{Al}, \mathrm{Ga}$, and $\mathrm{N}$, respectively, which were cited from Ref. 14 and incorporated with high-energylimit corrections. ${ }^{15}$ The intensities of both XRR patterns remarkably decrease from the glancing angle of $0.32^{\circ}$, which is in good agreement with the calculated critical angle of $0.323^{\circ}$. Consequently, the remarkable decrease in intensity of each XRR pattern is assigned to the change from the total reflection to the partial transmission, and the appearance of the oscillatory patterns in the range larger than $0.32^{\circ}$ is attributed to the interference originating from the multiple reflections in the $\mathrm{Al}_{0.2} \mathrm{Ga}_{0.8} \mathrm{~N}$ layer. In the range of $\theta>\theta_{c}$, the XRR intensity $I_{R}(\theta)$ is associated with the layer thickness $d$ through the following relation: ${ }^{16-18}$

$$
I_{R}(\theta) \propto \cos \left(2 \pi d \cdot \frac{2 \sqrt{\theta^{2}-\theta_{c}^{2}}}{\lambda}\right) .
$$

From the oscillatory patterns, we estimated the $\mathrm{Al}_{0.2} \mathrm{Ga}_{0.8} \mathrm{~N}$-layer thicknesses of Sample No. 1 and No. 2 in order to evaluate the reduction of the $\mathrm{Al}_{0.2} \mathrm{Ga}_{0.8} \mathrm{~N}$-layer thickness caused by the plasma exposure. The estimation of the $\mathrm{Al}_{0.2} \mathrm{Ga}_{0.8} \mathrm{~N}$-layer thickness was performed with the use of a Fourier transform (FT) method. ${ }^{17,18}$ In the FT procedure, the horizontal axis variant of the XRR pattern was transformed from $\theta$ to quasi-wavevector $\xi$ given by the following equation:

$$
\xi \equiv \frac{2 \sqrt{\theta^{2}-\theta_{c}^{2}}}{\lambda}
$$

In addition, the nonoscillatory portion of each XRR pattern was numerically subtracted, and the Hann window function was used so to suppress side robes. The FT power spectra of the oscillatory portions are depicted as a function of layer thickness in the inset of Fig. 1. From the peak of the FT band, the $\mathrm{Al}_{0.2} \mathrm{Ga}_{0.8} \mathrm{~N}$-layer thicknesses are estimated to be 25.8 and $22.6 \mathrm{~nm}$ for Sample No. 1 and No. 2, respectively. The reduction of the $\mathrm{Al}_{0.2} \mathrm{Ga}_{0.8} \mathrm{~N}$-layer thickness is about $12 \%$, which indicates that almost the whole $\mathrm{Al}_{0.2} \mathrm{Ga}_{0.8} \mathrm{~N}$ layer remains after the plasma exposure. The plasma exposure, however, remarkably modifies the decay of the XRR pattern as shown in Fig. 1. In the range of $\theta>\theta_{c}$, the decay of the XRR pattern of Sample No. 2 is larger than that of Sample No. 1. Since the XRR intensity is reduced by some surface roughness ${ }^{16,19}$ the decay profile of Sample No. 2 suggests that the surface damages causing the roughness are introduced by the plasma exposure.

In order to microscopically characterize the surface damages, the AFM measurement was performed. The AFM images of Sample No. 1 and No. 2 are shown in Figs. 2(a) and 2(b), respectively. The AFM image of Sample No. 1 manifests the atomic steps with clear boundaries. In addition, the dark spots, the so-called pits, are observed. The diameters of the pits are about $30 \mathrm{~nm}$. According to Refs. 20 and 21, the pits of the $\mathrm{Al}_{x} \mathrm{Ga}_{1-x} \mathrm{~N}$ surfaces are formed on specific threading dislocations, which hold a small fraction of the total dislocations, during the epitaxial growth. The specific dislocations are supposed to gather a large number of impurities at its core, which leads to the suppression of the layer growth at the core area and the subsequent formation of the pit. The AFM image of Sample No. 2, as shown in Fig. 2(b), clarifies that the following two changes emerge in the $\mathrm{Al}_{0.2} \mathrm{Ga}_{0.8} \mathrm{~N}$ surface after the plasma exposure. First, the boundaries of the atomic steps are blurred in comparison with those of Sample No. 1. Second, the diameters of the pits are increased to be about $50 \mathrm{~nm}$. The increase in diameter of the pit is relatively larger than the decrease in thickness of the $\mathrm{Al}_{0.2} \mathrm{Ga}_{0.8} \mathrm{~N}$ layer, which indicates that the region around the pit is selectively etched by the plasma exposure. From the above-mentioned findings, we conclude that the plasma exposure causes the surface roughness by selectively etching the atomic step boundaries and the pits. In general, the atomic step boundaries and pits lack completeness of a crystal such as continuity. We consider that the incompleteness of the boundaries and pits weakens the tolerance to the plasma exposure. In addition, as mentioned above, the microscopical compositions of the regions around the pits are different from those of the other region owing to the presence of impurities, which is another possible factor to reduce the tolerance to the plasma exposure.

\section{B. PL and reflectance spectra of the GaN layer in the $\mathrm{Al}_{0.2} \mathrm{Ga}_{0.8} \mathrm{~N} / \mathrm{GaN}$ heterostructures}

In advance to the PLE characterization, we performed the PL and reflectance measurements of the samples at $10 \mathrm{~K}$. 


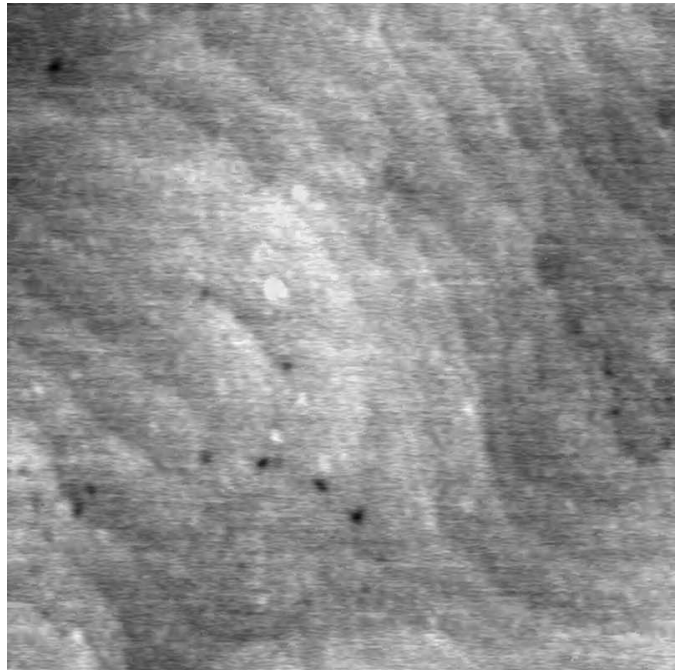

(a)

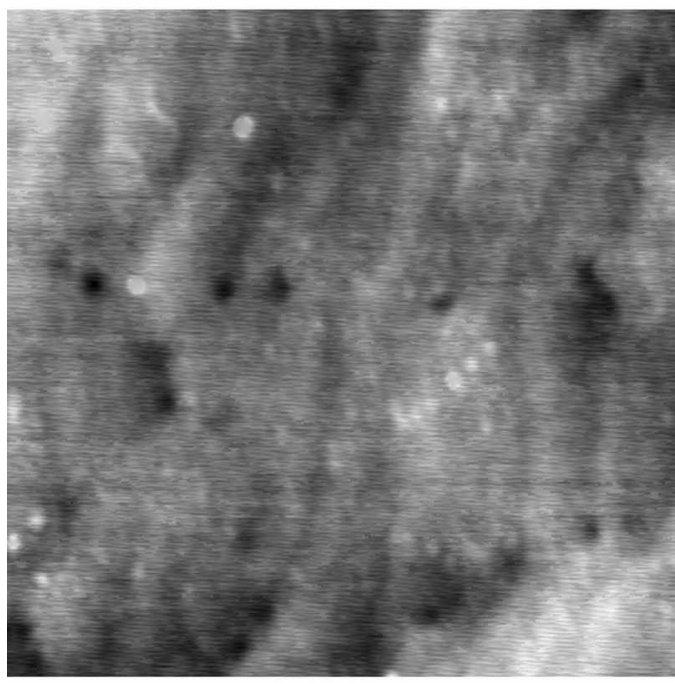

(b)

FIG. 2. AFM images of the $\mathrm{Al}_{02} \mathrm{Ga}_{0.8} \mathrm{~N}$ surfaces in the area of $1.0 \mu \mathrm{m}$ $\times 1.0 \mu \mathrm{m}$. (a) Sample No. 1. (b) Sample No. 2. Gray scale: height of 3.5 $\mathrm{nm}$ for (a), $2.5 \mathrm{~nm}$ for (b).

The PL and reflectance spectra of Sample No. 1 (the reference sample) and Sample No. 2 (the plasma-exposed one) are shown in Figs. 3(a) and 3(b), respectively, where the spectral range covers the energy range of the excitonic transition of GaN. The reflectance spectra exhibit the features peculiar to the excitonic transitions, which starts from the photon energy of $3.490 \mathrm{eV}$ toward the higher energy side. According to Ref. 22, the $A$ type exciton ( $A$ exciton) of GaN has the transition energy in units of $\mathrm{eV}$ given by the following equation at the temperature $T(\mathrm{~K})$ :

$$
E_{\mathrm{EX}, A}=-E_{\mathbf{b}, A}+3.503+\frac{5.08 \times 10^{-4} T^{2}}{T-996} .
$$

The quantity $E_{\mathbf{b}, A}$ is the binding energy of the $A$ exciton, value of which is $28 \mathrm{meV}$ for $\mathrm{GaN}^{22}$ From Eq. (5), the $A$ exciton transition energy is calculated to be $3.475 \mathrm{eV}$ at 10 $\mathrm{K}$. The starting energy of the excitonic features in the reflectance spectrum is higher by about $15 \mathrm{meV}$ than the expected $A$ exciton transition energy, which indicates that the exciton-
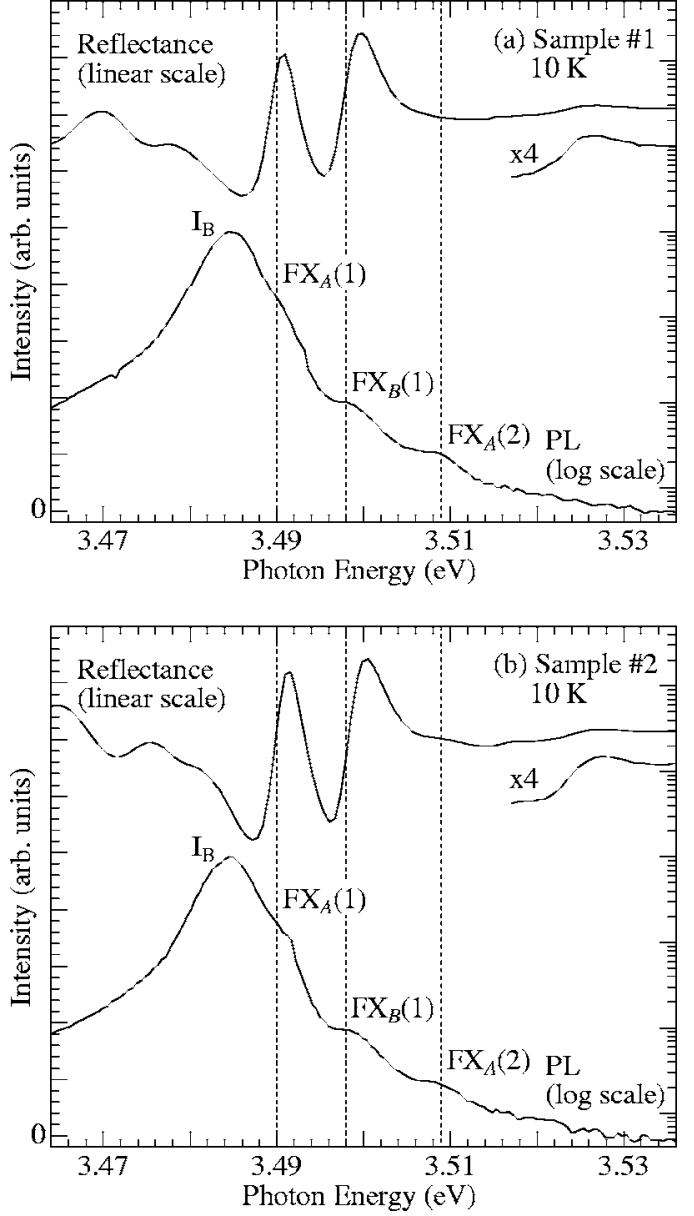

FIG. 3. PL (logarithmic scale) and reflectance (linear scale) spectra as a function of photon energy at 10 K. (a) Sample No. 1. (b) Sample No. 2. Dashed line: guide for the eyes.

transition energy in the present samples is raised by the presence of a compressive biaxial strain. The analysis of the strain effect will be described later.

The PL spectra of both the samples exhibit the dominant band labeled $\mathrm{I}_{\mathbf{B}}$ with a peak energy of $3.485 \mathrm{eV}$, which locates at the low energy side of the excitonic features appearing in the reflectance spectra. Accordingly, the origin of the peak at $3.485 \mathrm{eV}$ is assigned to the PL from the bound exciton of the $\mathrm{GaN}$ layer. The broad width of the band $\mathrm{I}_{\mathbf{B}}$, which is $5 \mathrm{meV}$, seems to mainly originate from inhomogeneous strains because a biaxial strain due to the epitaxial growth is not negligible: The strain effect will be discussed later with Fig. 5. In addition, the PL spectra of Sample No. 1 and No. 2 form the shoulders at 3.490, 3.498, and $3.509 \mathrm{eV}$, which are labeled $\mathrm{FX}_{A}(1), \mathrm{FX}_{B}(1)$, and $\mathrm{FX}_{A}(2)$, respectively. The energy range of the shoulders $\mathrm{FX}_{A}(1), \mathrm{FX}_{B}(1)$, and $\mathrm{FX}_{A}(2)$ is included within that of the excitonic features in the reflectance spectra, so that the origins of the shoulders $\mathrm{FX}_{A}(1)$, $\mathrm{FX}_{B}(1)$, and $\mathrm{FX}_{A}(2)$ are assigned to the PL bands from the free excitons of the GaN layer. Taking it into account that the $A$ exciton transition is the lowest in energy of the three kinds of excitonic transition in the GaN epitaxial layer under the compressive biaxial strain, ${ }^{23,24}$ the shoulder $\mathrm{FX}_{A}(1)$ is attributed to the PL from the ground-state $A$ exciton, the so-called $1 s$ state. 


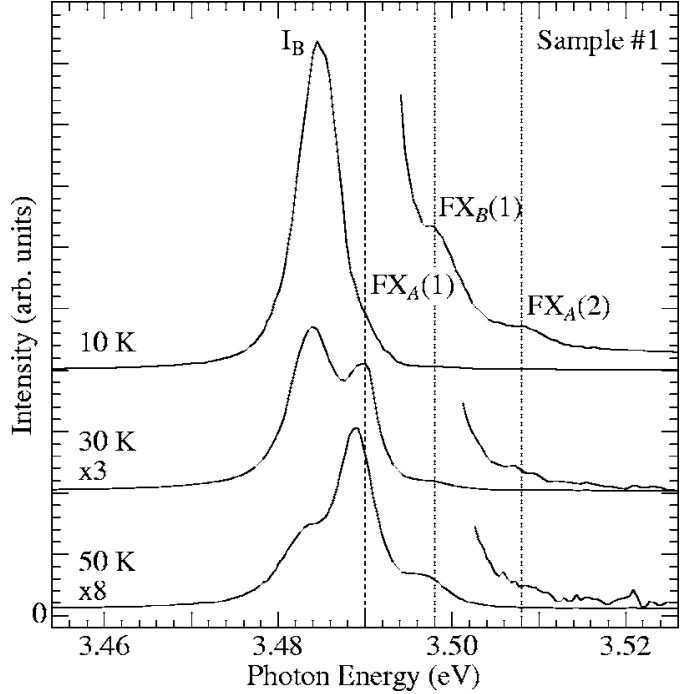

FIG. 4. PL spectra of Sample No. 1 at 10, 30, and $50 \mathrm{~K}$.

In general, bound excitons are thermally dissociated with an increase in temperature and, as a result, the dominant PL process changes from the bound exciton to the free exciton. Focusing on the general property of excitons, we additionally measured the PL spectra of Sample No. 1 at 30 and $50 \mathrm{~K}$, which are shown in Fig. 4 together with the PL spectrum at $10 \mathrm{~K}$, in order to confirm the assignment of the PL signals. It is obvious that the intensity of the PL band $\mathrm{I}_{\mathbf{B}}$ is remarkably reduced with increasing temperature. In contrast, the shoulder $\operatorname{FX}_{A}(1)$ becomes a dominant peak in addition to the fact that the shoulder $\mathrm{FX}_{B}(1)$ shows a clearer profile. The change of the dominant PL process coincides with the abovementioned general excitonic PL properties. Thus, it is confirmed that the spectral structures labeled $\mathrm{I}_{\mathbf{B}}$ and $\mathrm{FX}_{A}(1)$ result from the bound exciton and $A$ exciton, respectively. In the PL spectrum at $30 \mathrm{~K}$, the energy spacing between the bands $\mathrm{I}_{\mathbf{B}}$ and $\mathrm{FX}_{A}(1)$ is $5 \mathrm{meV}$, which is consistent with the result in Ref. 24. It is noted that the PL intensity integrated in the present energy range remarkably decreases with the increase in temperature, which suggests that most of the thermally activated excitons nonradiatively recombine through imperfections in the GaN layer. The decrease in the integrated PL intensity is responsible for the fact that we could not observe the PL corresponding to the shoulder $\mathrm{FX}_{A}(2)$ at $50 \mathrm{~K}$ owing to the detection limit of the apparatus.

The ground-state $A$ exciton transition energy of the present samples is larger by $15 \mathrm{meV}$ than that of unstrained GaN. From the energy shift of the $A$ exciton, we estimated the biaxial strain in the GaN layer and deduced other exciton transition energies. Figure 5 shows the shift of the exciton transition energy at the $\Gamma$ point, $\Delta E_{\mathrm{EX}, j}(n)$, calculated as a function of biaxial strain, where the subscript $j$ means the band of the hole forming the exciton and the index $n$ denotes the exciton principal quantum number. The calculation procedure consisted of the following three stages. At the first stage, the conduction-band energy $E_{\mathrm{CB}}$ and $j$-type valenceband energy $E_{\mathrm{VB}, j}$ at the $\Gamma$ point were calculated on the basis of the $\mathbf{k} \cdot \mathbf{p}$ perturbation theory under the quasicubic approximation proposed by Sirenko et al. ${ }^{25}$ The present calculations

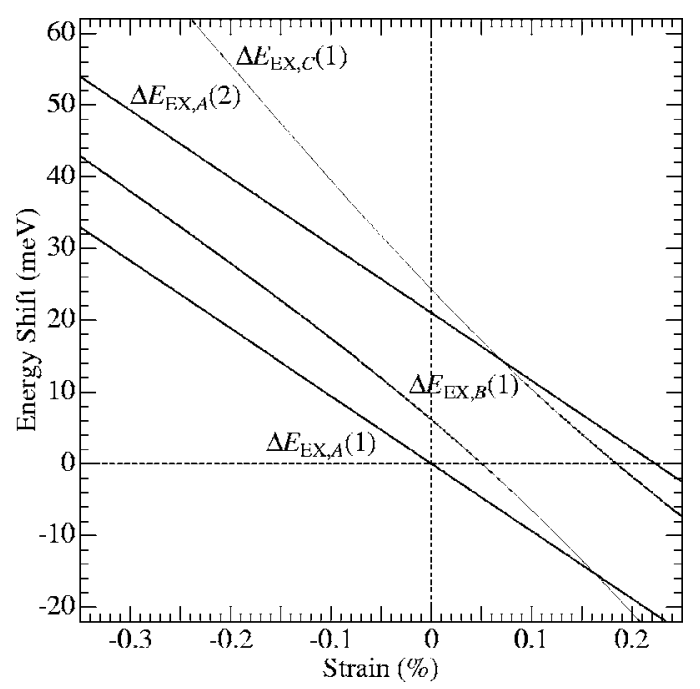

FIG. 5. Shift of the exciton transition energy at the $\Gamma$ point, $\Delta E_{\mathrm{EX}, j}(n)$, calculated as a function of biaxial strain. Subscript $j$ : band of the hole forming the exciton $(A, B$, or $C)$. Index $n$ : exciton principal quantum number $(n=1,2, \ldots)$.

of $E_{\mathrm{CB}}$ and $E_{\mathrm{VB}, j}$ utilized the parameters derived in Ref. 25. At the next stage, the exciton transition energies $E_{\mathrm{EX}, j}(n)$ were derived from the following relation:

$$
E_{\mathrm{EX}, j}(n)=E_{\mathrm{CB}}-E_{\mathrm{VB}, j}-\frac{1}{n^{2}} E_{\mathbf{b}, j}
$$

Here, the $B$ and $C$ exciton binding energies $E_{\mathbf{b}, B}$ and $E_{\mathbf{b}, C}$ were assumed to be equal to $E_{\mathbf{b}, A}$ for simplicity because the actual values of $E_{\mathbf{b}, A}, E_{\mathbf{b}, B}$, and $E_{\mathbf{b}, C}$ are still controversial. ${ }^{26}$ The present assumption is also employed in the theoretical study of optical transitions of GaN in Ref. 25. At the final stage, for convenience, the $A$ exciton transition energy with $n=1$ free from the strain, $E_{\mathrm{EX}, A, 0}(1)$, was chosen as the energy reference; namely

$$
\Delta E_{\mathrm{EX}, j}(n)=E_{\mathrm{EX}, j}(n)-E_{\mathrm{EX}, A, 0}(1) .
$$

In Fig. 5, the minus and plus signs of the biaxial strain correspond to the compression and dilatation, respectively. It can be read from Fig. 5 that the value of $15 \mathrm{meV}$ for $\Delta E_{\mathrm{EX}, A}(1)$ corresponds to the biaxial strain of $-0.16 \%$. This biaxial strain yields the values of 24,36 , and $49 \mathrm{meV}$ for $\Delta E_{\mathrm{EX}, B}(1), \Delta E_{\mathrm{EX}, A}(2)$, and $\Delta E_{\mathrm{EX}, C}(1)$, respectively. From the comparison with use of these values, the origins of the shoulders $\mathrm{FX}_{B}(1)$ and $\mathrm{FX}_{A}(2)$, which are shown in Fig. 3(b) as well as in Fig. 3(a), are assigned to the PL bands from the $B$ exciton with $n=1$ and $A$ exciton with $n=2$, respectively. In addition, the characteristic feature at $3.526 \mathrm{eV}$ in the reflectance spectra is attributed to the transition of the $C$ exciton with $n=1$.

It should be mentioned that PL spectra at a low temperature $(\sim 10 \mathrm{~K})$ are usually sensitive to the presence of imperfections. This is because the excitons bound to imperfections hardly escape from the bound states owing to a lack of the thermal activation energy; therefore, the PL from the free exciton is hardly observable. Taking account of the abovementioned PL properties of excitons, we discuss the PL spectra from the viewpoint of evaluating the effects of the plasma 

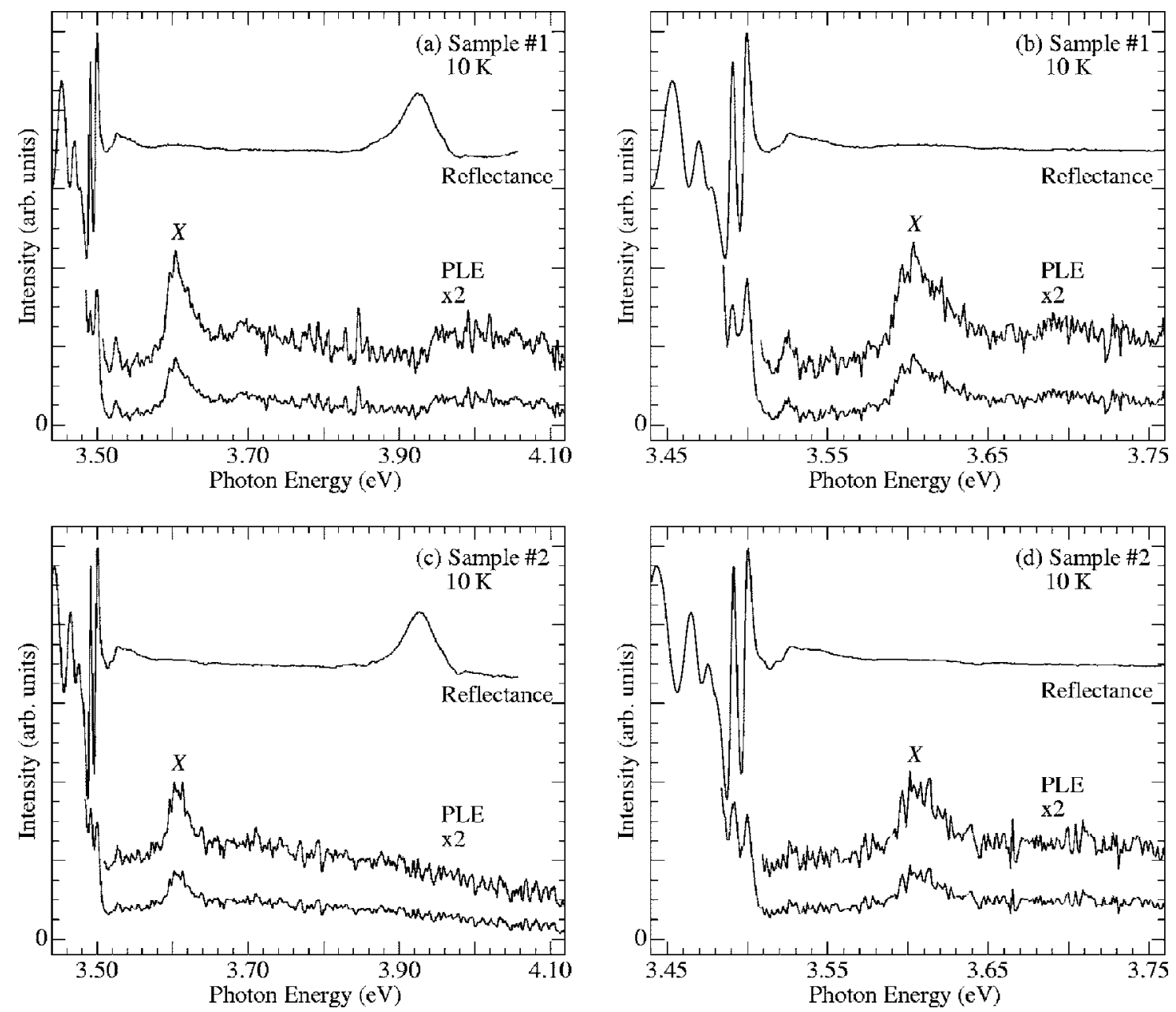

FIG. 6. PLE and reflectance spectra as a function of photon energy at $10 \mathrm{~K}$. Detection energy for the PLE spectra: $3.482 \mathrm{eV}$. (a) and (b) Sample No. 1. (c) and (d) Sample No. 2.

exposure on the GaN layer. As shown in Figs. 3(a) and 3(b), the shoulders $\operatorname{FX}_{A}(1), \operatorname{FX}_{B}(1)$, and $\mathrm{FX}_{A}(2)$, which originate from the free excitons, are clearly observed in Sample No. 2 as well as in Sample No. 1; namely, Sample No. 2, which was exposed to the plasma, shows the same PL profile as Sample No. 1. The present result demonstrates that the GaN layer of Sample No. 2 is the same in crystal quality as that of Sample No. 1, which reflects the fact that the plasma exposure does not introduce the imperfections to the GaN layer.

\section{Effects of the plasma exposure on the PLE characteristics of the $\mathrm{Al}_{0.2} \mathrm{Ga}_{0.8} \mathrm{~N}$ layer}

At first, we clarify the PLE and reflectance characteristics of Sample No. 1 that is the reference in the present work. The PLE spectrum of Sample No. 1 at $10 \mathrm{~K}$, which was measured at the detection energy of $3.482 \mathrm{eV}$ corresponding to the PL band $\mathrm{I}_{\mathbf{B}}$, is shown in Fig. 6(a), together with the reflectance spectrum. The PLE and reflectance spectra in the energy range from 3.45 to $3.75 \mathrm{eV}$ are also plotted in Fig. 6(b) in order to highlight the spectral profiles in this energy range. As shown in Fig. 6(b), the PLE spectrum exhibits the three narrow bands with a full width at half maximum of 8 meV, peaks of which locate at 3.491, 3.500, and $3.525 \mathrm{eV}$. According to the numerical calculation results presented in
Fig. 5, the peak energies agree with the transition energies of the $A, B$, and $C$ excitons with $n=1$ of the GaN layer in order of increasing energy; namely, the three narrow PLE bands originate from the photoabsorption due to the excitonic transitions. In addition to the three narrow bands, the PLE spectrum shows the broadband labeled $X$ with a peak energy of $3.61 \mathrm{eV}$ in the energy range between 3.59 and $3.64 \mathrm{eV}$. The appearance of the band $X$ indicates that the excitons with a specific kinetic energy efficiently relax to the bound exciton state. It is well known that the emission of longitudinaloptical (LO) phonons, which corresponds to LO-phonon scattering in momentum space, can promote the energy relaxation of the excitons. ${ }^{27}$ It should, however, be noted that the energy of the LO phonon with $E_{1}$ symmetry is $92 \mathrm{meV}$ for $\mathrm{GaN},{ }^{28}$ whereas the PLE band $X$ has an energy spacing of $125 \mathrm{meV}$ to the bound exciton PL band $\mathrm{I}_{\mathbf{B}}$ appearing at 3.485 $\mathrm{eV}$. The energy spacing of $125 \mathrm{meV}$ is fairly close to the AlN-like LO phonon energy; however, according to Ref. 29, the AlN-like optical phonon mode is missing in the Al content range lower than 0.6. In the present sample, the $\mathrm{Al}$ content is 0.2 ; therefore, the contribution of the AlN-like LO phonon can be ruled out. The exciton relaxation process related to the $X$ band seems to be phenomenologically explained as follows, taking account of the fact that the energy spacing of about $100 \mathrm{meV}$ between the $B$ exciton and the $X$ 
band is close to the LO-phonon energy of GaN. The exciton in the momentum space relaxes at first to the $B$-exciton state owing to the LO-phonon scattering, and then to the bound exciton state via the $A$-exciton state. Figure 6(b) also demonstrates that the PLE intensity is quite weak in the energy range higher than the fundamental exciton energies of $\mathrm{GaN}$, which indicates that the nonradiative recombination of electrons and holes photogenerated in the GaN layer is dominant.

The reflectance spectrum shown in Fig. 6(a) exhibits the prominent spectral feature with a peak energy of $3.93 \mathrm{eV}$ in the energy range between 3.82 and $3.98 \mathrm{eV}$, which corresponds to the excitonic transition energy of $\mathrm{Al}_{0.2} \mathrm{Ga}_{0.8} \mathrm{~N}$. The spectral feature at $3.93 \mathrm{eV}$ is much broader than the spectral features of the excitonic transitions of $\mathrm{GaN}$ ranging from 3.48 to $3.53 \mathrm{eV}$. The broadening of the excitonic transition profile of $\mathrm{Al}_{0.2} \mathrm{Ga}_{0.8} \mathrm{~N}$ is attributed to the electric field induced both by the piezoelectric polarization and by the spontaneous polarization in the $\mathrm{Al}_{0.2} \mathrm{Ga}_{0.8} \mathrm{~N}$ layer. ${ }^{30,31}$ It is well known that the electric field causes instability of the exciton. The presence of the electric field in the $\mathrm{Al}_{0.2} \mathrm{Ga}_{0.8} \mathrm{~N}$ layer has already been confirmed using the photoreflectance spectroscopy. ${ }^{32,33}$ The value of the electric field strength is estimated to be $215 \sim 230 \mathrm{kV} / \mathrm{cm}$ for the $27 \mathrm{~nm}$ thick $\mathrm{Al}_{0.2} \mathrm{Ga}_{0.8} \mathrm{~N}$ layers grown on the $\mathrm{GaN}$ buffer layers. The quantity $e F a_{\mathbf{B}}$, which is a measure of the electric field energy of the exciton with a Bohr radius $a_{\mathbf{B}}$ at the presence of the electric field strength $F$, is derived to be about $60 \mathrm{meV}$, where $a_{\mathbf{B}}$ is expressed using the reduced mass $\mu$ and binding energy $E_{\mathbf{b}}$ of the exciton ${ }^{34}$

$$
a_{\mathbf{B}}=\frac{\hbar}{\sqrt{2 \mu E_{\mathbf{b}}}} .
$$

The value of $\mu$ used is 0.191 in units of free electron mass, ${ }^{35}$ and the value of $E_{\mathbf{b}}$ is assumed to be the same as that of the exciton of $\mathrm{GaN}(28 \mathrm{meV})$. The value of $e F a_{\mathbf{B}}$ is about twice larger than the exciton binding energy, so that the two electric polarization components described above are responsible for the instability of the exciton in the $\mathrm{Al}_{0.2} \mathrm{Ga}_{0.8} \mathrm{~N}$ layer, which results in the spectral broadening. Moreover, a random alloy effect on the optical transition also broadens the reflectance signal of the $\mathrm{Al}_{0.2} \mathrm{Ga}_{0.8} \mathrm{~N}$ : the so-called alloy broadening.

As shown in Fig. 6(a), in regard to the excitonic transition of the $\mathrm{Al}_{0.2} \mathrm{Ga}_{0.8} \mathrm{~N}$ layer at $3.93 \mathrm{eV}$ in the reflectance spectrum, the step rising from $3.93 \mathrm{eV}$ toward the high energy side, which is usually attributed to the absorption profile of the fundamental transition-energy edge, is superimposed onto the PLE profile of the GaN layer. This indicates that the photogenerated carriers in the $\mathrm{Al}_{0.2} \mathrm{Ga}_{0.8} \mathrm{~N}$ layer flow into the GaN layer and contribute the PL from the bound exciton. We note that, in the energy range higher than the band edge of $\mathrm{Al}_{0.2} \mathrm{Ga}_{0.8} \mathrm{~N}$, the most of the excitation light still directly excites the GaN layer because the absorption coefficient of the $\mathrm{Al}_{0.2} \mathrm{Ga}_{0.8} \mathrm{~N}$ layer is deduced to be of the order of 1 $\times 10^{5} \mathrm{~cm}^{-1}$ according to Ref. 36 .

Next, we focus on the PLE and reflectance characteristics of Sample No. 2 that was exposed to the plasma. Figure 6(c) shows the PLE and reflectance spectra of Sample No. 2 at $10 \mathrm{~K}$, where the PLE-detection energy for Sample No. 2 is the same as that for Sample No. 1. For clarity, the PLE and reflectance spectra in the energy range of the excitonic transition of GaN are extracted and depicted in Fig. 6(d). For Sample No. 2, we could not observe a clear PLE profile of the $C$ exciton because of its relatively weak intensity. Except for this point, the PLE and reflectance characteristics of the GaN layer of Sample No. 2 are equivalent to those of Sample No. 1, which is consistent with the PL characteristics of Sample No. 1 and No. 2 shown in Figs. 3(a) and 3(b). Comparing the reflectance spectrum in Fig. 6(c) with that in Fig. $6(\mathrm{a})$, the reflectance characteristics of the $\mathrm{Al}_{0.2} \mathrm{Ga}_{0.8} \mathrm{~N}$ layer of Sample No. 2 are also the same as those of the $\mathrm{Al}_{0.2} \mathrm{Ga}_{0.8} \mathrm{~N}$ layer of Sample No. 1; namely, the reflectance spectrum of Sample No. 2 exhibits the same peak profile at $3.93 \mathrm{eV}$ as that of Sample No. 1, which is an obvious proof for the appearance of the excitonic transition. Thus, it is evident that the photoabsorption leading to the generation of carriers actually occurs in the $\mathrm{Al}_{0.2} \mathrm{Ga}_{0.8} \mathrm{~N}$ layer of Sample No. 2. In contrast to the reflectance characteristics, the drastic change appears in the PLE spectrum. The rising step corresponding to the absorption edge of the $\mathrm{Al}_{0.2} \mathrm{Ga}_{0.8} \mathrm{~N}$ layer disappears in the PLE spectrum of Sample No. 2. Instead of the rising step, the PLE spectrum shows the slope falling from $3.80 \mathrm{eV}$ toward the high energy side. The falling slope arises from the reduction of the total amount of photons absorbed in the $\mathrm{GaN}$ layer because of the light absorption in the top $\mathrm{Al}_{0.2} \mathrm{Ga}_{0.8} \mathrm{~N}$ layer. The PLE characteristics observed for Sample No. 2 indicate that the carriers generated through the photoabsorption in the $\mathrm{Al}_{0.2} \mathrm{Ga}_{0.8} \mathrm{~N}$ layer do not contribute to the bound exciton PL from the GaN layer. This is the key finding in the present work that highlights the difference in the spectroscopic nature between the PLE and reflectance characterizations. The reflectance characteristics, in principle, connect with the optical transitions at the critical points of the energy density of states, while the PLE signals reflect not only the optical transitions but also the carrier transport from a light-absorbing layer to a remote luminescent layer. The disappearance of the rising step is, therefore, attributed to the suppression of the carrier injection from the $\mathrm{Al}_{0.2} \mathrm{Ga}_{0.8} \mathrm{~N}$ layer to the $\mathrm{GaN}$ layer. According to the present structural characterizations described in Sec. III A, the plasma exposure introduces the damages to the atomic step boundaries and the regions around the pits on the surface of the $\mathrm{Al}_{0.2} \mathrm{Ga}_{0.8} \mathrm{~N}$ layer in Sample No. 2. The observed change in the PLE characteristics is the clear proof for the fact that the surface modification by the plasma exposure leads to the suppression of the carrier injection from the $\mathrm{Al}_{0.2} \mathrm{Ga}_{0.8} \mathrm{~N}$ layer to the $\mathrm{GaN}$ layer; namely, the surface recombination enhanced by the plasma exposure in the $\mathrm{Al}_{0.2} \mathrm{Ga}_{0.8} \mathrm{~N}$ layer is a major factor for preventing the carrier injection. Thus, it is concluded that the PLE characterization is applicable to assess the effects of the surface damages on the interlayer carrier transport. The conventional interpretation on the PLE characteristics, which relies only on the similarity between the absorption and PLE spectra, is liable to cause misunderstandings of the spectral analysis of semiconductor heterostructures. 


\section{SUMMARY}

We have systematically investigated the effects of the plasma-induced surface damages on the $\mathrm{Al}_{0.2} \mathrm{Ga}_{0.8} \mathrm{~N}(27 \mathrm{~nm}) / \mathrm{GaN}(2.2 \mu \mathrm{m})$ heterostructures using both the structural characterizations and the spectroscopic ones, where the AFM and XRR measurements were employed for the structural characterizations, and the PL, PLE, and reflectance measurements were utilized for the spectroscopic characterizations. Comparing the XRR pattern of the plasma-exposed sample with that of the as-grown reference sample, it has been revealed that the plasma exposure enhances the decay of the XRR pattern of the $\mathrm{Al}_{0.2} \mathrm{Ga}_{0.8} \mathrm{~N} / \mathrm{GaN}$ heterostructure. The enhancement of the decay indicates that the damages are introduced to the $\mathrm{Al}_{0.2} \mathrm{Ga}_{0.8} \mathrm{~N}$ surface. The AFM images have clarified the microscopical characteristics of the surface damages by the plasma exposure. The surface damages result from the selective etching both of the atomic step boundaries and of the pits, which suggests that the tolerance to the plasma exposure is decreased at the atomic step boundaries and pits. From the optical characterizations with use of the PL and reflectance measurements, it has been confirmed that the GaN layer of the plasma-exposed sample is almost the same in excitonic properties as the $\mathrm{GaN}$ layer of the reference sample; namely, the present plasma exposure does not modify the crystal quality of the GaN layer. In order to elucidate how the surface damages influence the optical properties of the $\mathrm{Al}_{0.2} \mathrm{Ga}_{0.8} \mathrm{~N}$ layer, the PLE characterization as well as the reflectance characterization have been applied both to the plasma-exposed sample and to the reference sample. The reflectance spectrum of the plasma-exposed sample shows the same pattern in the energy range of the $\mathrm{Al}_{0.2} \mathrm{Ga}_{0.8} \mathrm{~N}$ excitonic transitions as that of the reference sample; namely, the overall excitonic transition itself is not affected by the plasma exposure. In spite of the abovementioned fact, in the PLE spectra detected at the energy of the bound exciton PL from the underlying GaN layer, it has been found that the plasma exposure causes the disappearance of the rising step in the energy range above the fundamental transition energy of the $\mathrm{Al}_{0.2} \mathrm{Ga}_{0.8} \mathrm{~N}$ layer. This demonstrates that, in the plasma-exposed sample, the photogenerated carriers in the $\mathrm{Al}_{0.2} \mathrm{Ga}_{0.8} \mathrm{~N}$ layer cannot contribute to the PL from the bound exciton in the GaN layer. We, therefore, conclude that the PLE measurement can be applied to probe the interlayer photogenerated-carrier transport in the $\mathrm{Al}_{x} \mathrm{Ga}_{1-x} \mathrm{~N} / \mathrm{GaN}$ heterostructures. The present findings also warn that the optical characterization based only on the similarity between the absorption and PLE spectra leads to misinterpretation of the PLE signals.

\section{ACKNOWLEDGMENTS}

One of authors, M.N., thanks the financial support by the Grant-in-Aid for Creative Scientific Research from Japan Society for the Promotion of Science (No. 17GS1204).
${ }^{1}$ For a review, H. H. Wieder, Surf. Sci. 132, 390 (1983).

${ }^{2}$ For a review, D. E. Aspnes, Surf. Sci. 132, 406 (1983).

${ }^{3}$ T. Saitoh and H. Hasegawa, Appl. Surf. Sci. 56-58, 94 (1992).

${ }^{4}$ S. Anantathanasarn and H. Hasegawa, J. Vac. Sci. Technol. B 19, 1589 (2001).

${ }^{5}$ J.-Y. Duboz and M. Asif Khan, in Group III Nitride Semiconductor Compounds: Physics and Applications, edited by B. Gil (Clarendon Press, Oxford, 1998), Chap. 9.

${ }^{6}$ S. Karmalkar, M. S. Shur, and R. Gaska, in Wide Energy Bandgap Electronic Devices, edited by F. Ren and J. C. Zolper (World Scientific Publishing, Singapore, 2003), Chap. 3

${ }^{7}$ Y. Zhou, R. Chu, J. Liu, K. J. Chen, and K. M. Lau, Phys. Status Solidi C 2, 2663 (2005).

${ }^{8}$ T. Kikkawa, M. Nagahara, N. Okamoto, Y. Tateno, Y. Yamaguchi, N. Hara, K. Joshin, and P. M. Asbeck, in Technical Digest of 2001 International Electron Devices Meeting (IEEE, Piscataway, 2001), pp.585-588.

${ }^{9}$ O. Mitrofanov, M. Manfra, and N. Weimann, Appl. Phys. Lett. 82, 4361 (2003).

${ }^{10}$ C. F. Klingshirn, Semiconductor Optics (Springer-Verlag, Berlin, 1997), Chap. 24

${ }^{11}$ D. K. G. de Boer, A. J. G. Leenaers, and W. W. van den Hoogenhof, X-Ray Spectrom. 24, 91 (1995).

${ }^{12}$ J. J. Song and W. Shan, in Group III Nitride Semiconductor Compounds: Physics and Applications, edited by B. Gil (Clarendon Press, Oxford, 1998), Chap. 6.

${ }^{13}$ V. W. L. Chin, T. L. Tansley, and T. Osotchan, J. Appl. Phys. 75, 7365 (1994).

${ }^{14}$ D. T. Cromer and D. Liberman, J. Chem. Phys. 53, 1891 (1970).

${ }^{15}$ L. Kissel and R. H. Pratt, Acta Crystallogr. A 46, 170 (1990).

${ }^{16}$ L. G. Parratt, Phys. Rev. 95, 359 (1954).

${ }^{17}$ K. Sakurai and A. Iida, Jpn. J. Appl. Phys., Part 2 31, L113 (1992).

${ }^{18}$ K. Sakurai and A. Iida, Adv. X-Ray Anal. 35, 813 (1992).

${ }^{19}$ S. K. Sinha, E. B. Sirota, S. Garoff, and H. B. Stanley, Phys. Rev. B 38, 2297 (1988).

${ }^{20}$ Z. Liliental-Weber, Y. Chen, S. Ruvimov, and J. Washburn, Phys. Rev. Lett. 79, 2835 (1997).

${ }^{21}$ Y. Chen, T. Takeuchi, H. Amano, I. Akasaki, N. Yamada, Y. Kaneko, and S. Y. Wang, Appl. Phys. Lett. 72, 710 (1998).

${ }^{22}$ B. Monemar, Phys. Rev. B 10, 676 (1974).

${ }^{23}$ B. Gil, F. Hamdani, and H. Morkoç, Phys. Rev. B 54, 7678 (1996).

${ }^{24}$ W. Shan, R. J. Hauenstein, A. J. Fischer, J. J. Song, W. G. Perry, M. D. Bremser, R. F. Davis, and B. Goldenberg, Phys. Rev. B 54, 13460 (1996).

${ }^{25}$ Y. M. Sirenko, J. B. Jeon, B. C. Lee, K. W. Kim, M. A. Littlejohn, M. A. Stroscio, and G. J. Iafrate, Phys. Rev. B 55, 4360 (1997).

${ }^{26}$ I. Vurgaftman and J. R. Meyer, J. Appl. Phys. 94, 3675 (2003).

${ }^{27}$ M. Umlauff, J. Hoffmann, H. Kalt, W. Langbein, J. M. Hvam, M. Scholl, J. Söllner, M. Heuken, B. Jobst, and D. Hommel, Phys. Rev. B 57, 1390 (1998).

${ }^{28}$ A. Cingolani, M. Ferrara, M. Lugarà, and G. Scamarcio, Solid State Commun. 58, 823 (1986)

${ }^{29}$ V. Yu. Davydov, I. N. Goncharuk, A. N. Smirnov, A. E. Nikolaev, W. V. Lundin, A. S. Usikov, A. A. Klochikhin, J. Aderhold, J. Graul, O. Semchinova, and H. Harima, Phys. Rev. B 65, 125203 (2002).

${ }^{30}$ For a review, E. T. Yu, X. Z. Dang, P. M. Asbeck, S. S. Lau, and G. J. Sullivan, J. Vac. Sci. Technol. B 17, 1742 (1999).

${ }^{31}$ O. Ambacher, J. Smart, J. R. Shealy, N. G. Weimann, K. Chu, M. Murphy, W. J. Schaff, L. F. Eastman, R. Dimitrov, L. Wittmer, M. Stutzmann, W. Rieger, and J. Hilsenbeck, J. Appl. Phys. 85, 3222 (1999).

${ }^{32}$ H. Takeuchi, Y. Yamamoto, Y. Kamo, T. Oku, and M. Nakayama, Eur. Phys. J. B 52, 311 (2006)

${ }^{33}$ H. Takeuchi, Y. Yamamoto, Y. Kamo, T. Kunii, T. Oku, S. Wakaiki, and M. Nakayama, Eur. Phys. J. Appl. Phys. 37, 119 (2007).

${ }^{34}$ P. K. Basu, Theory of Optical Processes in Semiconductors (Clarendon Press, Oxford, 1997), p. 126.

${ }^{35}$ M. Suzuki, T. Uenoyama, and A. Yanase, Phys. Rev. B 52, 8132 (1995).

${ }^{36}$ J. F. Muth, J. D. Brown, M. A. L. Johnson, Z. Yu, R. M. Kolbas, J. W. Cook, Jr., and J. F. Schetzina, MRS Internet J. Nitride Semicond. Res. 4S1, G5.2 (1999). 